\section{PTU-130 REGULATORY T CELLS IN ACUTE LIVER FAILURE ARE FUNCTIONALLY INTACT AND MAY CONTRIBUTE TO RETENTION OF NEUTROPHILS IN AREAS OF HEPATIC NECROSIS}

${ }^{1} \mathrm{R}$ Chudasama*, ${ }^{1} \mathrm{Y}-\mathrm{Y}$ Chen, ${ }^{1} \mathrm{H}$ Jeffery, ${ }^{1} \mathrm{C}$ Thomas, ${ }^{2} \mathrm{~N}$ Murphy, ${ }^{2} \mathrm{~T}$ Whitehouse, ${ }^{1} \mathrm{DH}$ Adams, ${ }^{1} \mathrm{YH}$ Oo. ${ }^{1}$ Centre for Liver Research and NIHR BRU, University of Birmingham, UK; ${ }^{2}$ Intensive Care Unit, UHB NHS Foundation Trust, Birmingham, UK

\subsection{6/gutjnl-2014-307263.204}

Introduction Acute liver failure (ALF) is a sterile inflammation with a high mortality. The immunological response to acute liver injury is initiated by the infiltration of innate neutrophils and is followed by an adaptive T cells response. T cells in drug induced skin lesions have been shown to express neutrophil chemoattractant and there is also evidence that regulatory $\mathrm{T}$ cells (Tregs) in peripheral blood secrete IL-8. However, little is known on the ability of liver infiltrating $\mathrm{T}$ cell subsets to retain neutrophils in ALF or the function of Tregs. Our aim is to investigate the phenotypic features and cytokine profiles of circulating and liver infiltrating $\mathrm{T}$ cell subsets from ALF patients with a view of assessing their functional ability to retain neutrophils.

Methods Distribution and localisation of Liver infiltrating lymphocytes and neutrophils were assessed by immunohistochemistry. Peripheral blood and liver infiltrating lymphocytes were isolated from ALF patients. Intracellular-cytokines expression profiles of the lymphocytes subsets were assessed by flow cytometry. Functional status of T cells including Tregs was assessed by pSTAT5 signalling.

Results Immunohistochemistry revealed high numbers of neutrophils in ALF compared to chronic diseased livers $(109 \pm 12.2$ vs. $7.1 \pm 2.67 ; \mathrm{p}=0.01)$ and normal liver $(109 \pm 12.2$ vs. $0.8 \pm$ $0.33 ; \mathrm{p}=0.0002)$. Dual immunohistochemistry showed co-localisation of lymphocytes and neutrophils in areas of hepatic necrosis. Neutrophil chemoattractant IL-8 expression in peripheral blood was higher in lymphocyte subsets of ALF patients compared to normal donor, $\mathrm{CD} 3(2.3 \pm 0.54 \%$ vs. $0.93 \pm 0.4 \% ; \mathrm{p}=0.23)$, $\mathrm{CD} 4$ $(2.8 \pm 2.2 \%$ vs. $1.1 \pm 0.7 \% ; \mathrm{p}=0.41), \mathrm{CD} 8(2.2 \pm 1 \%$ vs. 0.61 $\pm 0.09 \% ; \mathrm{p}=0.43)$ and Treg $(0.8 \pm 0.4 \%$ vs. $0.2 \pm 0.02 \% ; \mathrm{p}=$ $0.48)$. There was an up-regulation of IL-8 production in Tregs after 7 days of ex vivo expansion compared to day zero $(1.63 \pm 1 \%$ vs. $0.77 \pm 0.42 \% ; p=0.44)$. IFN- $\gamma$ expression in ALF peripheral blood compared with normal blood was $(23.1 \pm 6.2 \%$ vs. $11.1 \pm$ $8.4 \% ; \mathrm{p}=0.38)$ for CD3 $(11.72 \pm 2.3 \%$ vs. $7.8 \pm 6.26 ; \mathrm{p}=0.5)$ for CD4 and $(14.21 \pm 3.04$ vs. $9.95 \pm 8.44 ; \mathrm{p}=0.58)$ for CD8 after day seven of ex vivo expression. Importantly, Tregs from both blood and explanted liver of ALF were functional indicated by STAT5 phosphorylation in response to IL-2.

Conclusion We demonstrated for the first time that lymphocyte subsets including Tregs in ALF produce IL-8, which may contribute to the retention of neutrophils in areas of hepatic necrosis in ALF. We also showed that Tregs and other T cells are functionally responsive to IL-2 in both blood and explanted liver tissue of ALF patients. Disclosure of Interest None Declared.

\section{PTU-131 PREVALENCE OF ABNORMAL LIVER FUNCTIONS TESTS (LFTS) IN OBSTRUCTIVE SLEEP APNEA (OSA) PATIENTS AND CHANGE IN LFTS AFTER CONTINUOUS POSITIVE AIRWAY PRESSURE (CPAP) THERAPY}

\footnotetext{
1,2R Haider*, ${ }^{2} \mathrm{HJ}$ O'Connor, ${ }^{2,3} \mathrm{M}$ Azam. 'Gastroenterology, TAGG, Dublin; ${ }^{2}$ Gastroenterology, Naas Hospital, Naas, Ireland; ${ }^{3}$ Gastroenterology, Sunderland Royal Hospital, Sunderland, UK
}

Introduction Obstructive Sleep Apnea (OSA) is a recurrent obstruction of the upper airways during sleep leading to intermittent hypoxia $(\mathrm{IH})$. OSA is associated with metabolic syndrome and with non-alcoholic fatty liver disease with abnormal liver function tests (LFTs).

Aims We looked at prevalence of abnormal LFTs in OSA patients and effects of CPAP therapy on LFTs.

Methods In this retrospective study we did chart review of 100 patients of OSA who were commenced on CPAP from 2008 to 2013. We noted their age, body mass index (BMI), alcohol intake status, LFTs 3-18 months before and after CPAP therapy, ultrasound findings and their cholesterol levels.

Results Patient's were in the age group of 38-82 with average (ave) age of 57.5 years. There were 24\% Women and $76 \%$ were men. Ave BMI was 36.4. Epworth S improved from 12.7 to 5.2 after CPAP therapy. Apnea Hypopnea Index improved from 36.17 to 4.52. A total of 59 of 100 OSA patients had abnormal LFTs at the start of CPAP therapy as compared to $35 \%$ patients after therapy which included either abnormal Alanine transaminase (ALT), Gamma-glutamyltransferase (GGT) or Alkaline phosphatase or in combination which suggests significant improvement. There were 10 patients with newly abnormal LFTs after therapy. Prior to CPAP 45 of 100 patients had abnormal ALT with average ALT of 58. 27 of 45 patients had ALT improvement. Ave ALT post therapy was 28.18 of 45 patients had no improvement and there were 4 new patients with abnormal ALT at the end of study. 51 of 100 patients had abnormal GGT and 33 of 51 had improvement post therapy. 18 patients either had worsening or no improvement and there were 6 new patients with abnormal GGT at the end of study. 10 of 100 patients had abnormal Alk p and only 2 had improvement and 8 had no improvement Bilirubin levels of all patients were normal. 37\% had fatty liver reported on abdominal ultrasounds. 15 patients had alcohol intake history including patients with occasional intake, Worsening of LFTs noticed in this group. BMI reduced in 7 patients. 5 of 7 patients had LFTs improvement in this group. Remaining patients BMI fluctuated by $1 \mathrm{~kg}$ over study period. 52\% had high cholesterol. Of 52 patients, 14 patients had improvement in cholesterol and 7 patients had LFTs improved in this group. 8 Patients did not comply with CPAP. There was no change in LFTS in 6 patients with 1 patient it improved and in 1 they worsened. Conclusion The study showed that overall prevalence of patients with abnormal LFTs was high in OSA patients (i.e. 59\%) and there was significant improvement in ALT and GGT as compared to ALK $\mathrm{p}$ after CPAP therapy. Study suggests that optimal assessment and treatment of OSA may lead to LFTs improvement and it demands further prospective studies.

Disclosure of Interest R. Haider Employee of: Trinity College, H. O'Connor Consultant for: Clinical Professor in Gastroenterology, M. Azam Consultant for: Gastroenterology.

\section{PTU-132 HEPATOLOGY E-CONSULTATION: THE WAY FORWARD? A REVIEW OF OUR INITIAL EXPERIENCE AND COST ANALYSIS}

'S Moreea*, 'PB Southern, ${ }^{2} \mathrm{~J}$ Connolly, 'VJ Appleby. 'Gastroenterology, Digestive Diseases Centre, Bradford Royal Infirmary, Duckworth Lane; ${ }^{2}$ Bradford and Airedale Primary Care Trust, Bradford, UK

10.1136/gutjnl-2014-307263.206 